\title{
Strong Thermopower Enhancement and Tunable Power Factor via Semimetal to Semiconductor Transition in a Transition-Metal Dichalcogenide
}

\author{
Hongjae Moon ${ }^{1}$, Joonho Bang ${ }^{2}$, Seokkyoon Hong ${ }^{1}$, Gwansik Kim ${ }^{1}$, Jong Wook Roh ${ }^{3}$, \\ Jeongmin $\mathrm{Kim}^{1, \mathrm{a})}$, and Wooyoung Lee ${ }^{1, \mathrm{a})}$ \\ ${ }^{1}$ Department of Materials Science and Engineering, Yonsei University, 50 Yonsei-ro, \\ Seodaemoon-gu, Seoul, 03722, Republic of Korea \\ ${ }^{2}$ Department of Energy Science, Sungkyunkwan University, Suwon 16419, Republic \\ of Korea \\ ${ }^{3}$ School of Nano and Materials Science and Engineering, Kyungpook National \\ University, Gyeongsangbuk-do 37224, Republic of Korea
}

\section{Supporting Information}

\footnotetext{
a) Authors to whom any correspondence should be addressed.

(E-mail: wooyoung@yonsei.ac.kr and jmkim@yonsei.ac.kr)
} 


\section{DFT first-principles calculations of electronic band structures and DOS}
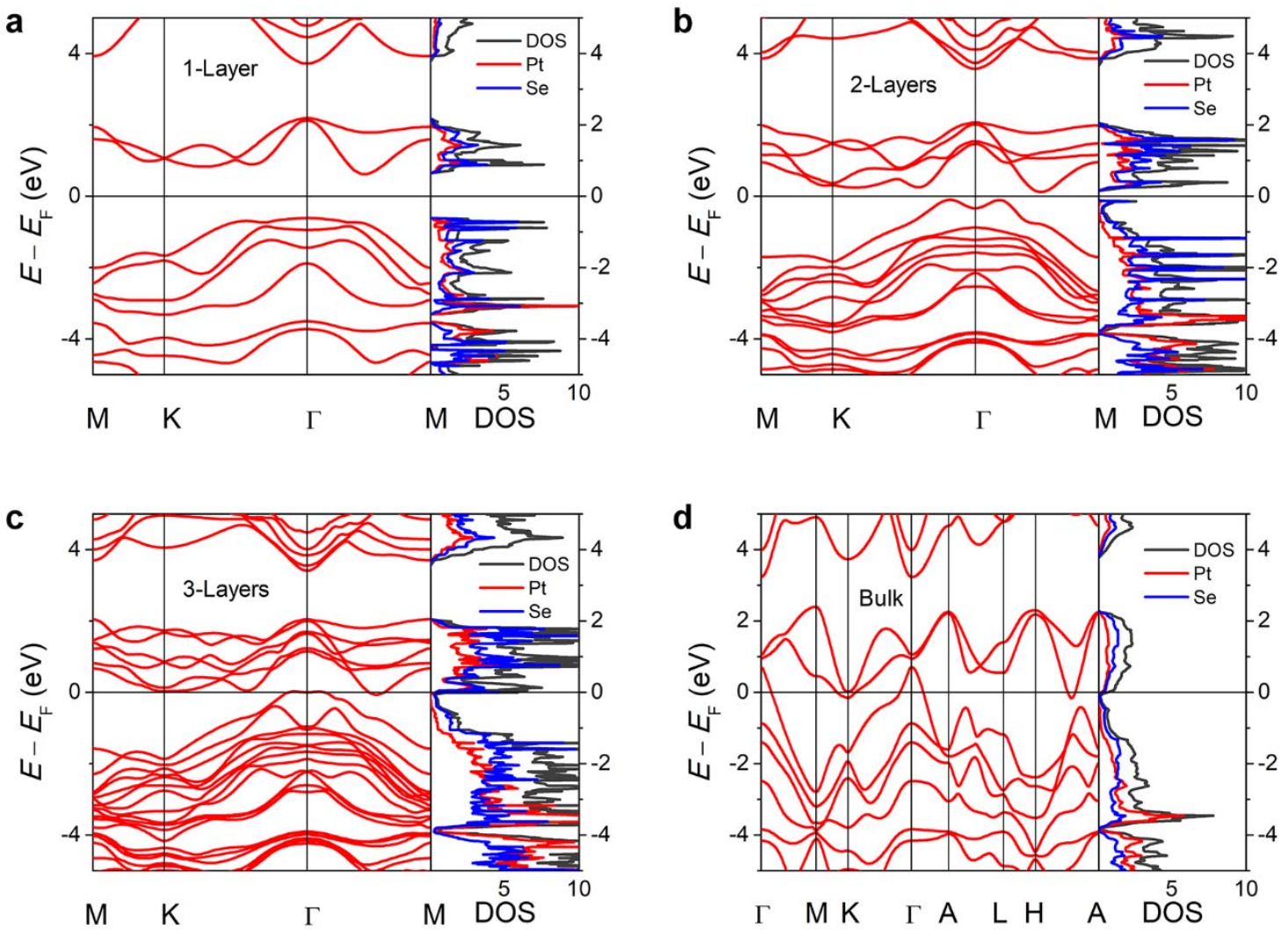

Figure S1. DFT first-principles calculations of the electronic band structures and DOS of $\mathrm{PtSe}_{2}$ of different thicknesses. (a) Monolayer nanosheets. (b) Bilayer nanosheets. (c) Trilayer nanosheets. (d) Bulk.

The monolayer and bilayer nanosheets have a semiconducting band structure with bandgaps of 1.23 and $0.23 \mathrm{eV}$, respectively. The band structure of the trilayer nanosheet and bulk crystal indicate that they are semimetals with band overlap energies of 0.09 and $0.87 \mathrm{eV}$, respectively. 


\section{BTE calculations of transport properties as a function of energy}

a

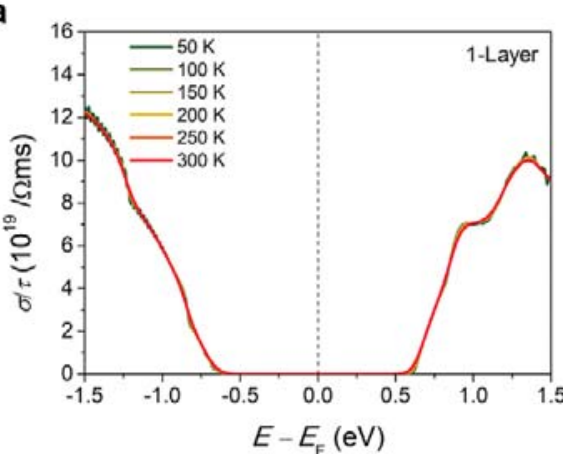

b
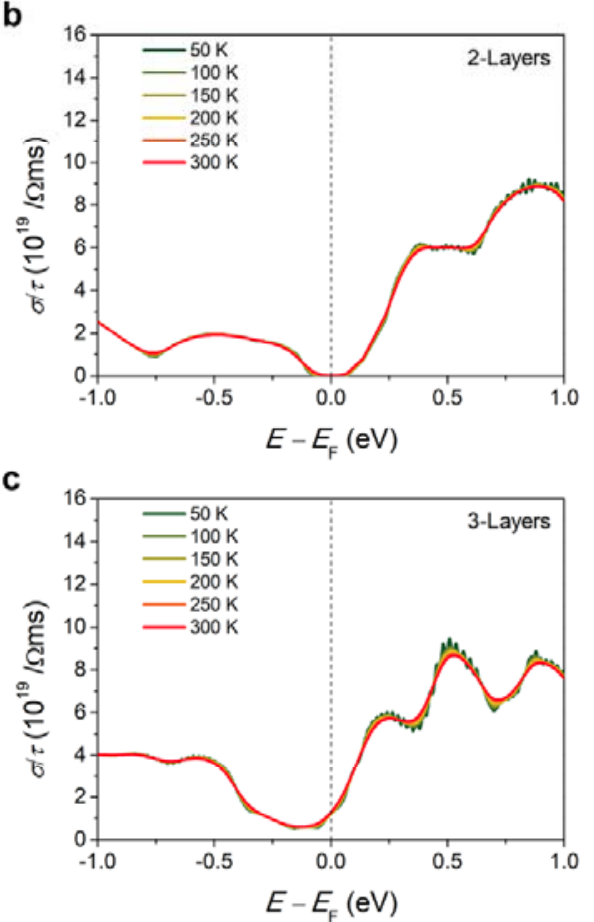

d

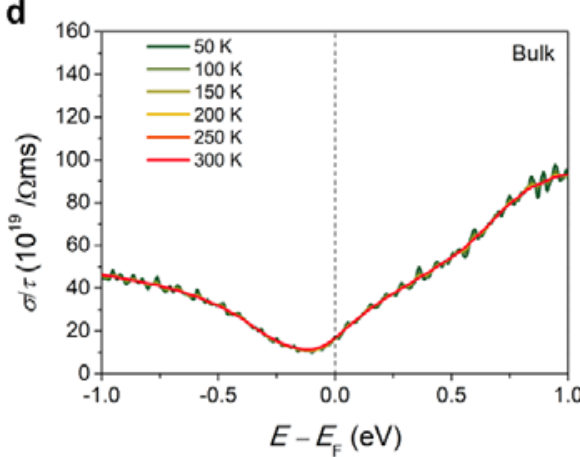

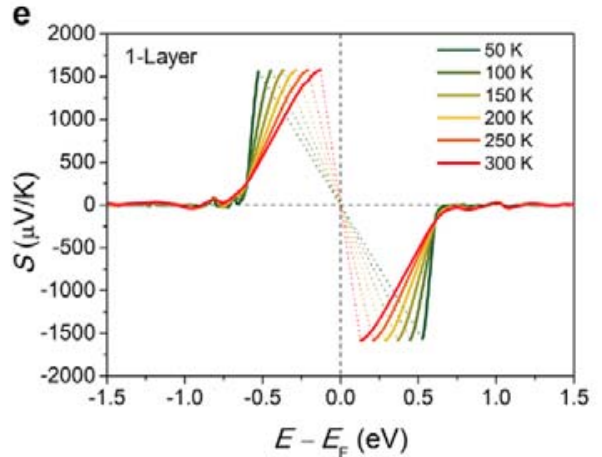

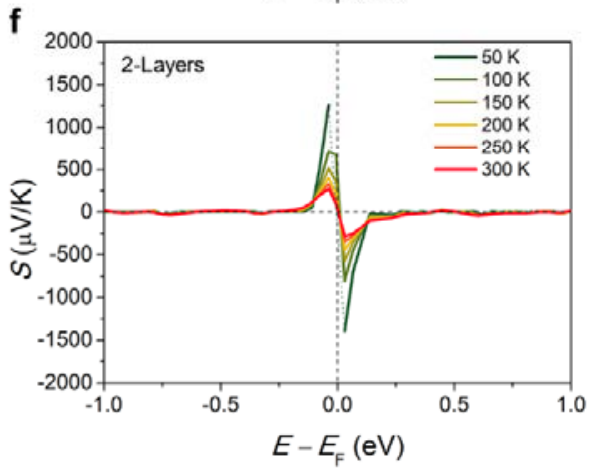

g

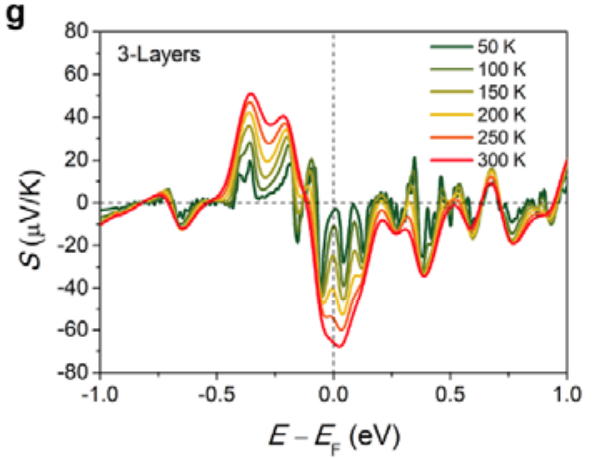

h

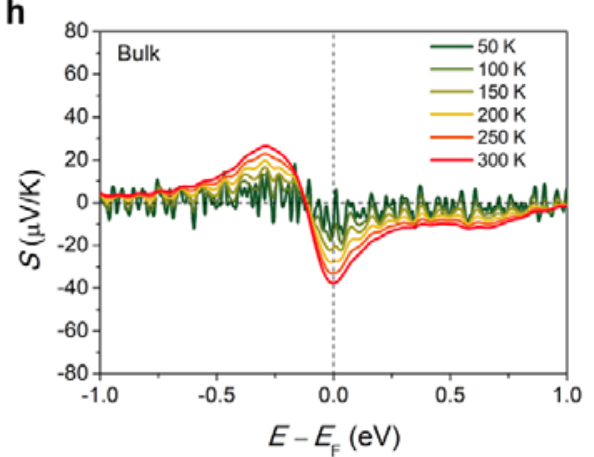

Figure S2. Predicted transport properties of $\mathrm{PtSe}_{2}$ of different thicknesses as a function of energy obtained by solving the Boltzmann transport equation based on the DFT calculations. (a, b, c, d) Electrical conductivity $(\sigma)$ with respect to scattering time $(\tau)$ at different temperatures for monolayer nanosheets (a), bilayer nanosheets (b), trilayer nanosheets (c) and bulk (d). (e, f, $\mathrm{g}, \mathrm{h})$ Thermopower (S) at different temperatures for monolayer nanosheets (e), bilayer nanosheets (f), trilayer nanosheets (g) and bulk (h). 


\section{BTE calculations of transport properties as a function of carrier density}
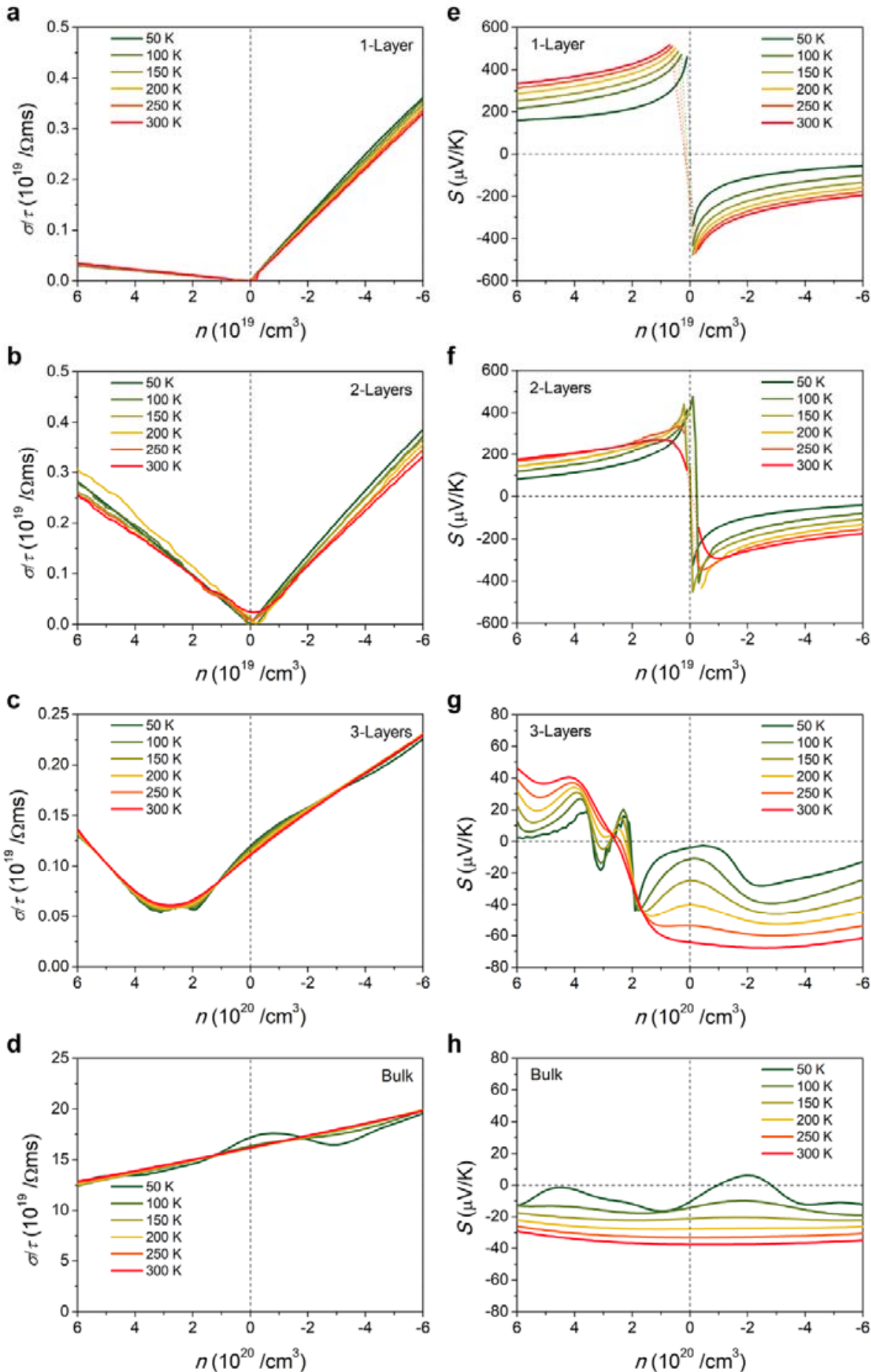

Figure S3. Predicted transport properties of $\mathrm{PtSe}_{2}$ of different thicknesses as a function of carrier density $(n)$ obtained by solving the Boltzmann transport equation based on the DFT calculations. (a, b, c, d) Electrical conductivity $(\sigma)$ with respect to scattering time $(\tau)$ at different temperatures for monolayer nanosheets (a), bilayer nanosheets (b), trilayer nanosheets (c) and bulk (d). (e, f, g, h) Thermopower $(S)$ at different temperatures for monolayer nanosheets (e), bilayer nanosheets (f), trilayer nanosheets $(\mathrm{g})$ and bulk $(\mathrm{h})$. 


\section{Measurement techniques and errors}
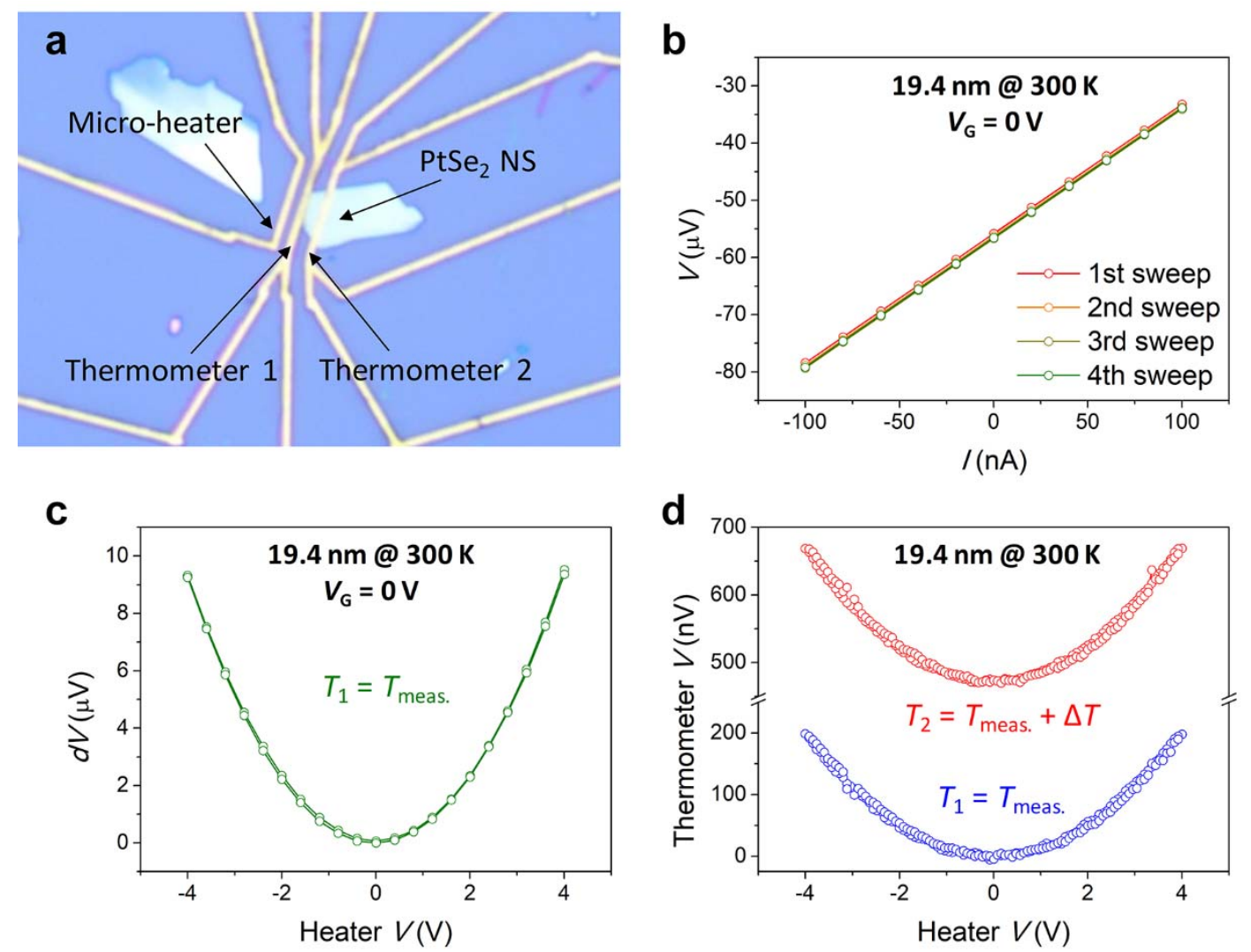

Figure S4. Device and measurement techniques for transport characterizations. (a) Micro device for electrical conductivity and thermopower measurements. (b) $I-V$ curves for resistance measurement. (c) Thermopower voltages and (d) thermometer voltages measured as a function of the heater voltage.

In the electrical conductivity measurements, $I-V$ curves were obtained using two electrodes (thermometer 1 and 2 ) to obtain the resistance $(R)$. The electrical conductivity was calculated by the relation, $\sigma=(R w t / L)^{-1}$, where $w, t$, and $L$ are the width, thickness, and channel length of the nanosheet, respectively. Since the $I-V$ curves were linear and reproducible, the error of resistance measurement was negligible. Therefore, the errors in electrical conductivity were dominated by the uncertainty of the nanosheet thickness measured by the AFM height profile, and thus we were able to obtain the error of electrical conductivity based on the standard deviation of multiple thickness profiling.

In the thermopower measurement, a temperature gradient was generated by Joule heating using the micro-heater to produce as thermopower voltage $(d V)$. The thermopower voltage between the two thermometers was proportional to the square of the heater voltage applied to the microheater, following the heating power $P=V^{2} / R$. The temperature difference was measured using the resistance increase of each thermometer, which also shows a quadratic relationship with the heater voltage. The resistance of the thermometers was measured as a voltage signal, which was obtained by a four-probe method (each thermometer has four electrodes). To calibrate the 
temperature-voltage ratio for each thermometer, the measurement was performed at two different ambient temperatures (for the $300 \mathrm{~K}$ measurement, $T_{1}=300 \mathrm{~K}$ and $T_{2}=305 \mathrm{~K}$ ). Finally, the thermopower $(S)$ was calculated by dividing the thermopower voltage by the temperature difference between two thermometers. In this thermopower measurement, the reliability was confirmed by the clear parabolas shown by the thermopower and thermometer voltages, and the thermopower value and error were obtained from the repeated sweeps of the heater voltage using the obtained thermopower values at the highest heater voltage, because measurement error decreases as increasing the temperature difference (Four thermopower measurements are conducted in one heater voltage sweep).
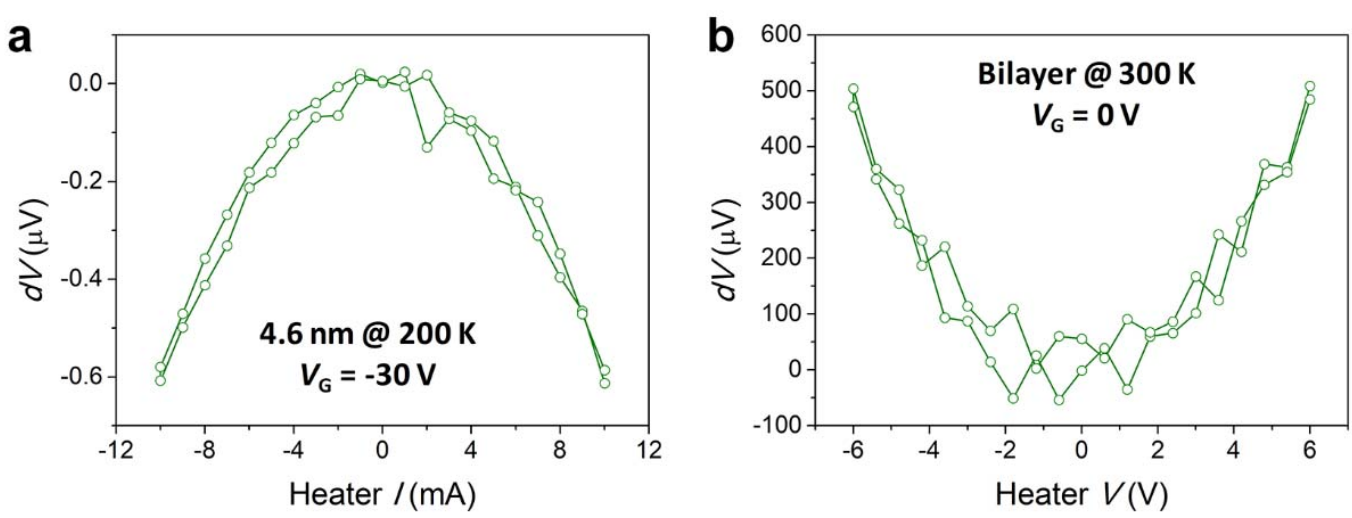

Figure S5. Representative raw data with small signal to noise ratio. (a) Raw data for the smallest thermopower value. (b) Raw data for the thermopower with largest error.

In the thermopower measurements, generally, the noise of thermopower voltage is smaller than that of thermometer voltages. Therefore, the most measurement errors originated from the temperature calibration using thermometer voltages. Although the decrease in the thermopower voltage causes an increase in error due to a decrease in the signal to noise ratio, the absolute value of the error is not large because the thermopower value is small. However, near the off state where the thermopower values are very large, the reduced signal to noise increases the measurement errors. 

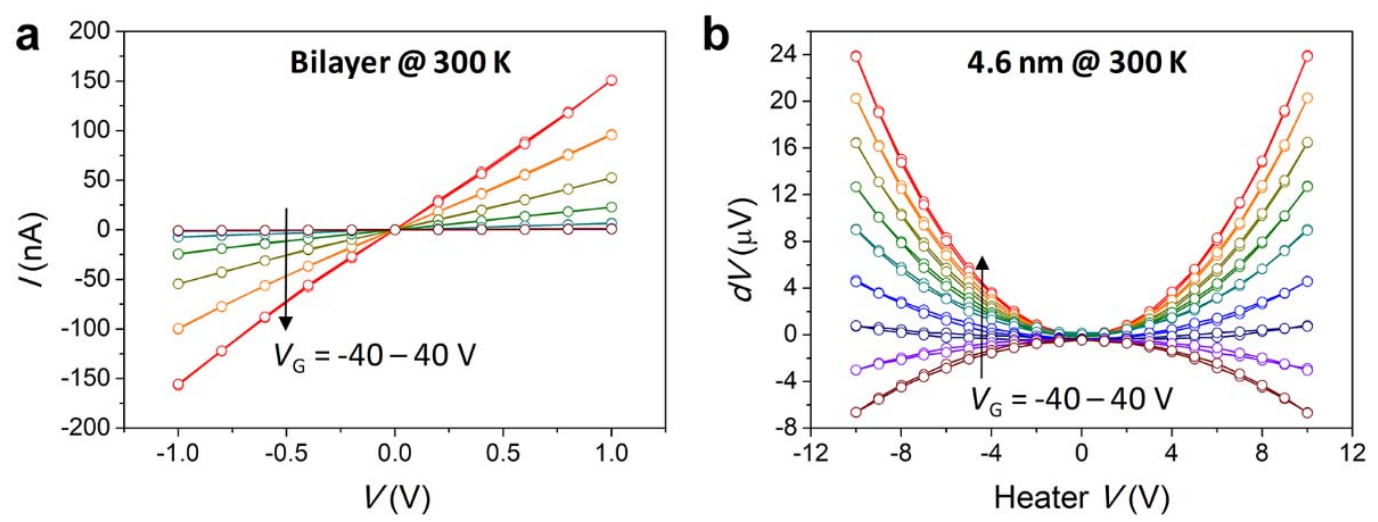

Figure S6. Representative raw data for gate dependent transport properties. (a) $I-V$ curves for the bilayer $\mathrm{PtSe}_{2}$ nanosheet in -40 to $40 \mathrm{~V}$ gate voltage range. (b) Thermopower voltages for $4.6 \mathrm{~nm} \mathrm{PtSe} 2$ nanosheet in -40 to $40 \mathrm{~V}$ gate voltage range.

The $I-V$ curves are almost linear even in the bilayer semiconducting nanosheet, and the thermopower voltages show clear parabolic shape as a function of the heater voltage. 


\section{Stability and reproducibility of $\mathrm{PtSe}_{2}$ nanosheets}
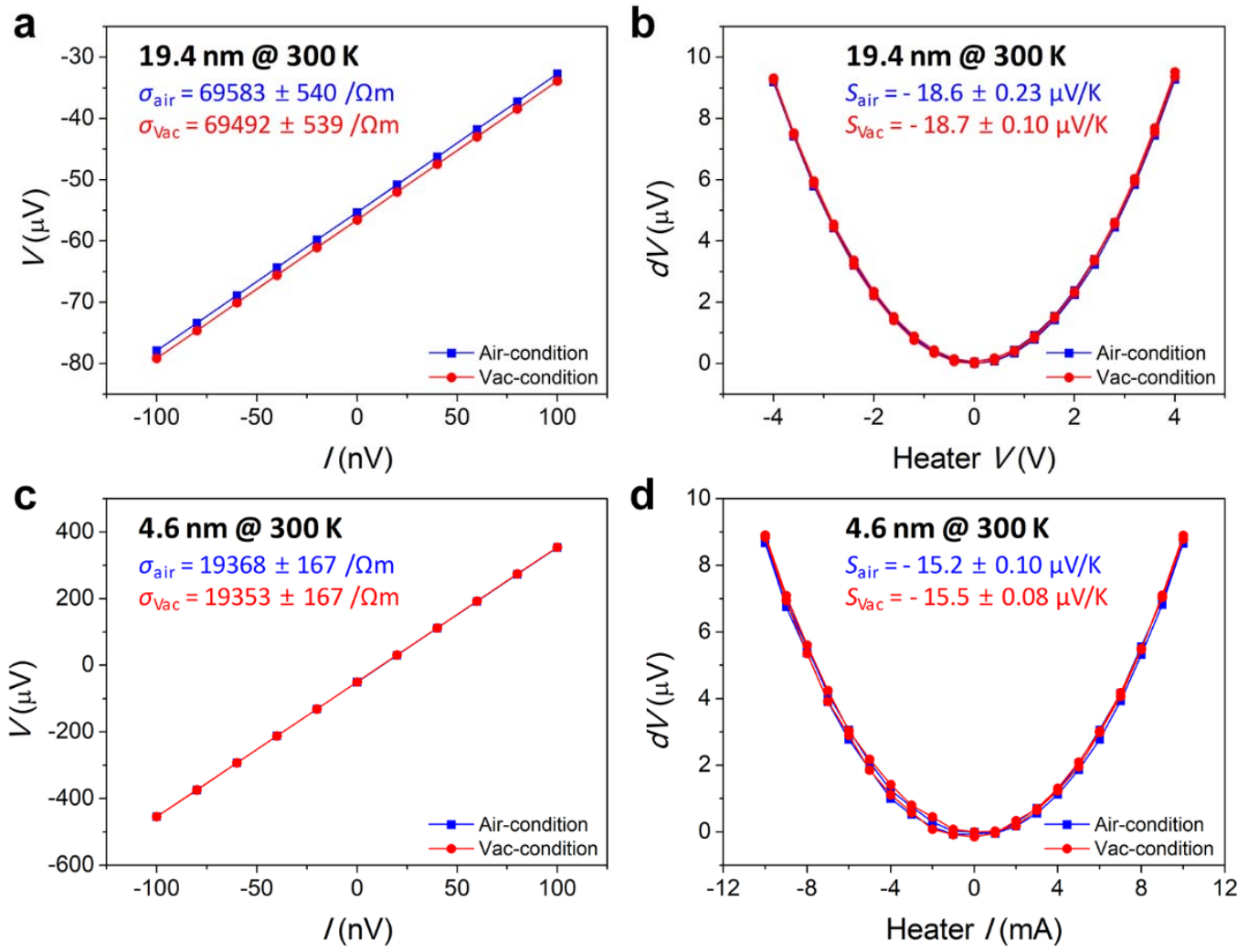

Figure S7. Transport properties of 19.4 and $4.6 \mathrm{~nm}$ nanosheets before (blue squares) and after (red circles) vacuum at room temperature. (a, c) Electrical conductivity $(\sigma)$ values and raw data ( $I-V$ curve). The slopes (resistance) of $I-V$ curves are the same within the error range. $(\mathrm{b}, \mathrm{d})$ Thermopower $(S)$ values and raw data (thermopower voltages).

The slight difference in the thermopower is attributed to the stabilization of the ambient temperature. The transport properties measured in air-condition remain almost constant after being stored in high vacuum. 

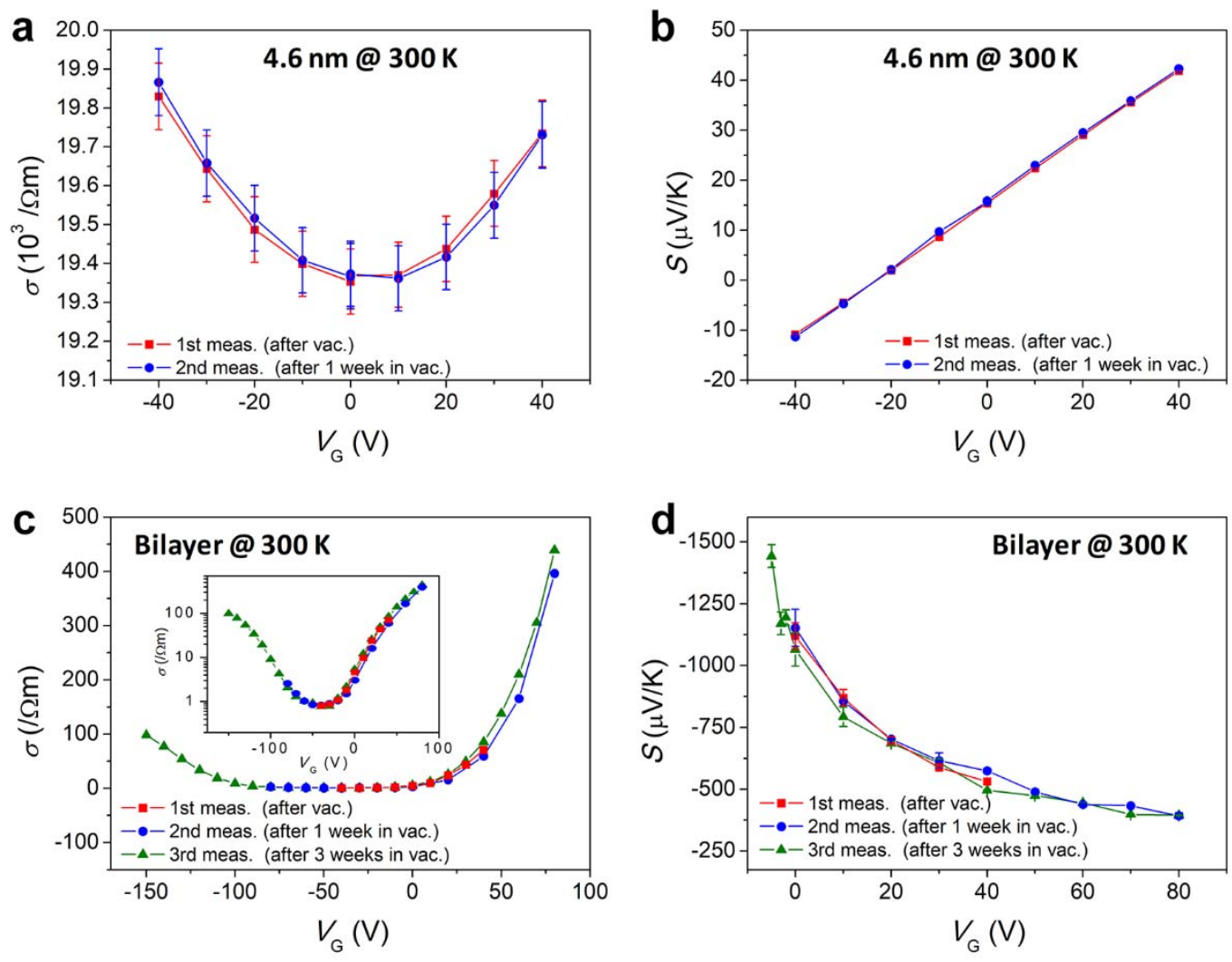

Figure S8. Transport properties of $4.6 \mathrm{~nm}$ and bilayer nanosheets after 1 day (red squares), 1 week (blue circles), and 3 weeks (green triangles) in vacuum at room temperature. (a, c) Gated electrical conductivity. $(b, d)$ Gated thermopower.

Multiple measurements with temperature and electric field cycling were performed as gradually increasing the gate voltage sweep ranges, which demonstrated the stability of $\mathrm{PtSe}_{2}$ nanosheets and reproducibility of devices. 


\section{Transport characterization of $40 \mathrm{~nm}$ nanosheet}

a

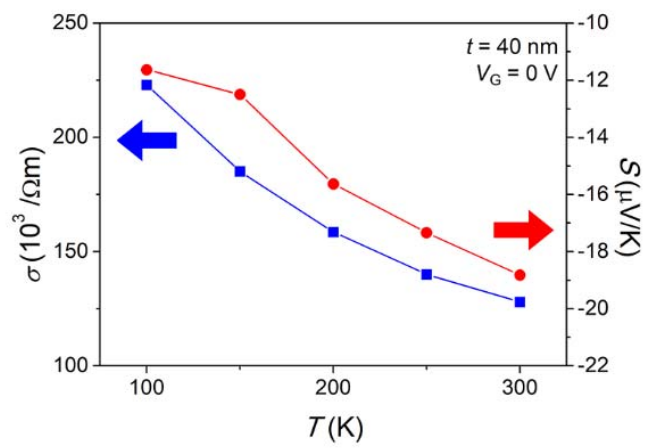

b

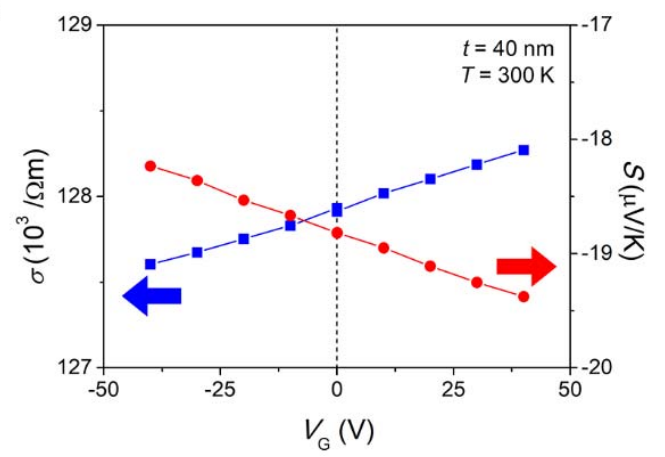

Figure S9. Transport characterization of a metal-like PtSe2 nanosheet of a thickness of $40 \mathrm{~nm}$. (a) Temperature dependent electrical conductivity ( $\sigma$, blue squares) and thermopower $(S$, red circles) at zero gate voltage. (b) Gate-modulated electrical conductivity ( $\sigma$, blue squares) and thermopower ( $S$, red circles) at room temperature.

The temperature dependence of the transport properties and the very weak gate dependence show the metal-like transport characteristics of the 40-nm $\mathrm{PtSe}_{2}$ nanosheet. 


\section{Simple two band model in semimetals}
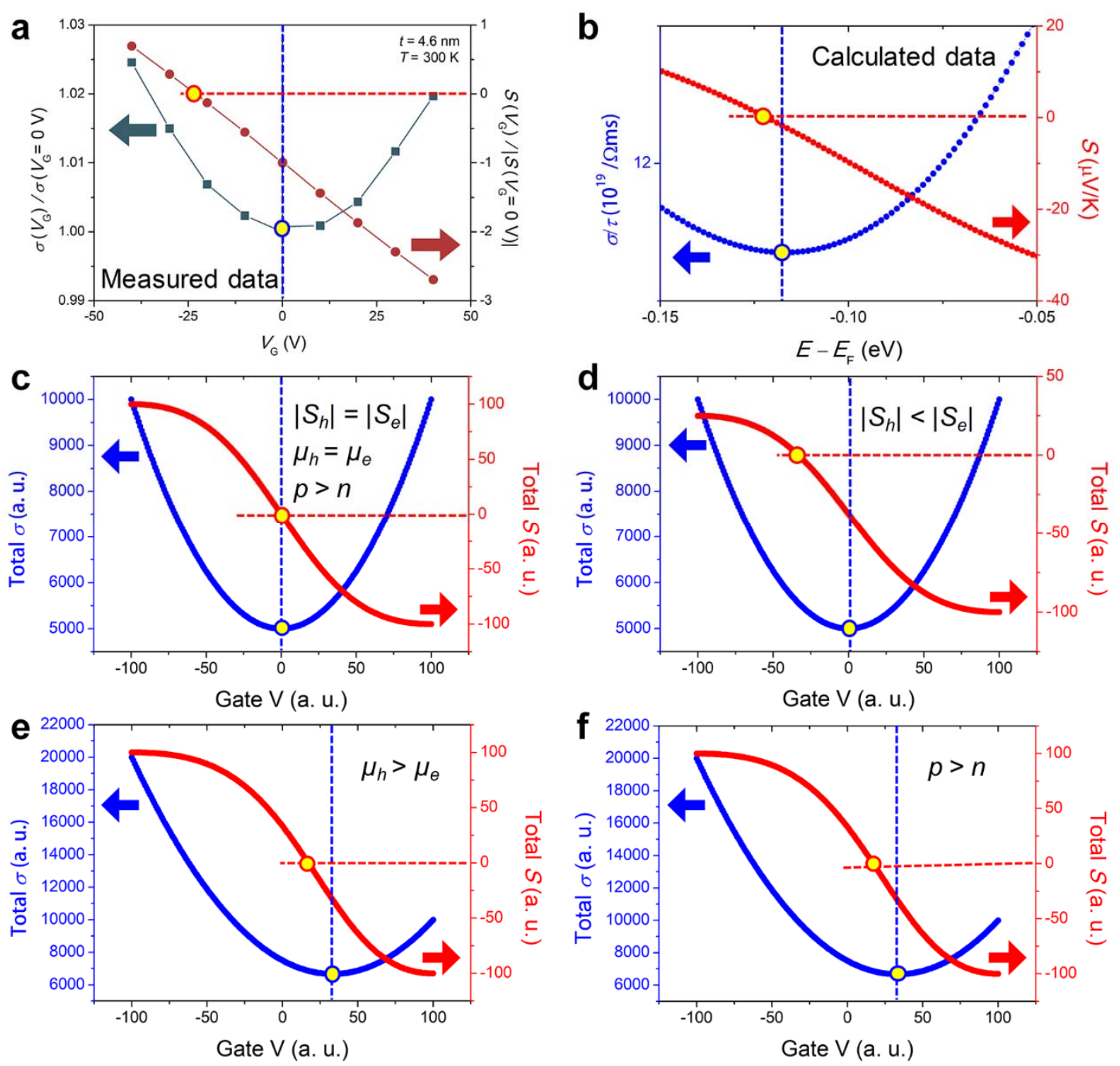

Figure S10. Experimental and theoretical transport characterizations. (a) Normalized electrical conductivity (dark blue squares) and thermopower (red circles) with respect to the gate voltage of the thick nanosheet at room temperature (Fig. 3h). (b) Predicted electrical (blue) conductivity and thermopower (red) obtained by solving the Boltzmann transport equation based on the DFT calculations (Fig. S2d and h). (c-f) Calculated electrical conductivity (blue) and thermopower (red) based on simple two bands model.

Intrinsic properties of valence and conduction bands are the same (c). Partial thermopower of valence band is smaller than that of conduction band (d). mobility (e) or carrier density (f) of valence band is larger than that of conduction band. In semimetals where holes and electrons coexist, the total transport properties can be understood by the simple two band model. ${ }^{1-2}$ In this model, the total electrical conductivity $\left(\sigma_{\text {total }}\right)$ and total thermopower $\left(S_{\text {total }}\right)$ are given by $\sigma_{\text {total }}=\sigma_{h}+\sigma_{e}$ and $S_{\text {total }}=\left(\sigma_{h} S_{h}+\sigma_{e} S_{e}\right) /\left(\sigma_{h}+\sigma_{e}\right)$, where the subscripts $h$ and $e$ refer to holes and electrons, respectively. Since the total thermopower is determined by the compensation of partial thermopower weighted by the partial conductivity of holes and 
electrons, the equivalent points of the two properties can be different in semimetals. When the intrinsic properties and bands for holes (valence band) and electrons (conduction band) are similar, the transport properties changes symmetrically with the variation of carrier density (c). But, changes in intrinsic properties cause loss of symmetry and differences in equivalent points (d, e, and f). The calculations based on the Boltzmann transport equation were also consistent with the measured results (b). 


\section{Contact resistance and 4-terminal devices}
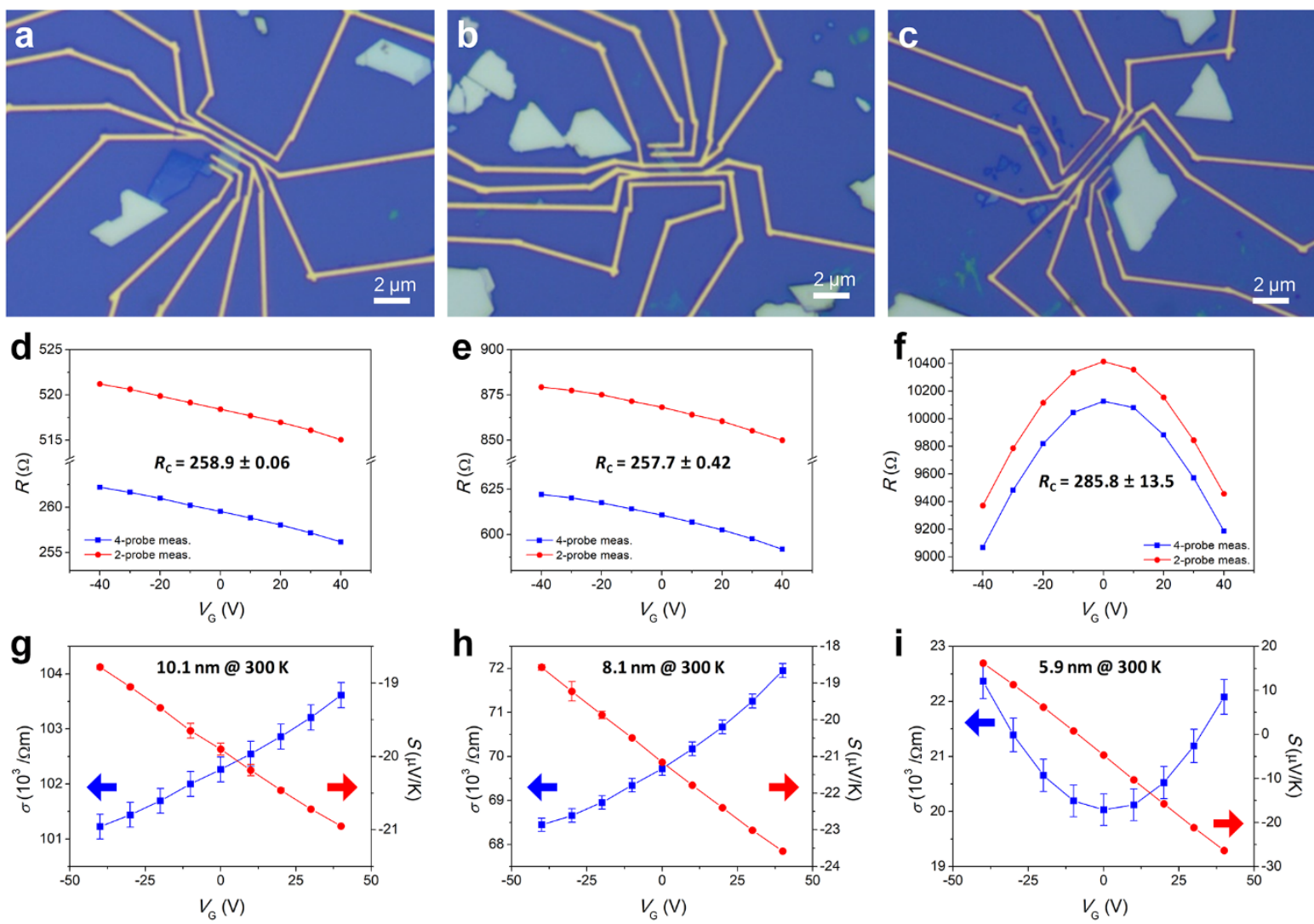

Figure S11. Characterization of contact resistance and 4-terminal devices based on $\mathrm{PtSe}_{2}$ nanosheets with 10.1, 8.1, and $5.9 \mathrm{~nm}$. (a, b, c) Micro devices for the 4-probe measurement to estimate the contact resistance. (d, e, f) Gated 4- and 2-probe resistances and contact resistances at room temperature. $(\mathrm{g}, \mathrm{h}, \mathrm{i})$ Gated electrical conductivity obtained from 4-probe measurement and gated thermopower in 10.1, 8.1, and $5.9 \mathrm{~nm}$ nanosheets at room temperature.

The contact resistance $\left(R_{\mathrm{C}}\right)$ between the nanosheet and electrodes was investigated to obtain accurate power factor. The 4-terminal devices were fabricated using 10.1, 8.1, and $5.9 \mathrm{~nm}$ nanosheet to determine and exclude the contact resistance based on the 4-probe measurement technique. The obtained contact resistances were less than $300 \Omega$ even in the semimetallic nanosheet without gate-dependence, and the deviation in electrical conductivity were negligible in the semimetallic nanosheets. In the case of semiconducting nanosheets, however, it is difficult to obtain the contact resistance using the 4-probe method, because the $V-I$ measurements are required owing to the high resistance and off state. The deviation in electrical conductivity due to the contact resistance is noticeable in the metal-like nanosheets. The gated electrical conductivity and thermopower of 10.1, 8.1, and $5.9 \mathrm{~nm}$ nanosheets are in good agreement with the thickness dependence of gated transport properties obtained from 2terminal devices. 


\section{References}

1. Hicks, L. D.; Dresselhaus, M. S., Effect of Quantum-Well Structures on the Thermoelectric Figure of Merit. Phys. Rev. B 1993, 47, 12727-12731.

2. Kim, J.; Lee, S.; Brovman, Y. M.; Kim, P.; Lee, W., Diameter-Dependent Thermoelectric Figure of Merit in Single-Crystalline Bi Nanowires. Nanoscale 2015, 7, 50535059. 